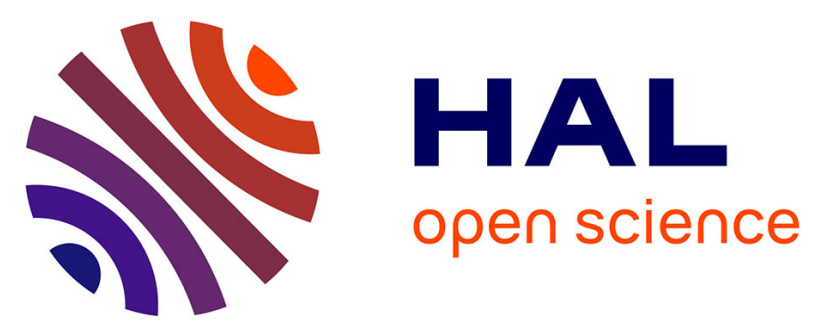

\title{
Adaptive Trajectory Tracking for Quadrotor MAVs in Presence of Parameter Uncertainties and External Disturbances
}

Gianluca Antonelli, Elisabetta Cataldi, Filippo Arrichiello, Paolo Robuffo Giordano, Stefano Chiaverini, Antonio Franchi

\section{To cite this version:}

Gianluca Antonelli, Elisabetta Cataldi, Filippo Arrichiello, Paolo Robuffo Giordano, Stefano Chiaverini, et al.. Adaptive Trajectory Tracking for Quadrotor MAVs in Presence of Parameter Uncertainties and External Disturbances. IEEE Transactions on Control Systems Technology, 2018, 26 (1), pp.248-254. 10.1109/TCST.2017.2650679 . hal-01483653

\section{HAL Id: hal-01483653 \\ https://hal.inria.fr/hal-01483653}

Submitted on 6 Mar 2017

HAL is a multi-disciplinary open access archive for the deposit and dissemination of scientific research documents, whether they are published or not. The documents may come from teaching and research institutions in France or abroad, or from public or private research centers.
L'archive ouverte pluridisciplinaire HAL, est destinée au dépôt et à la diffusion de documents scientifiques de niveau recherche, publiés ou non, émanant des établissements d'enseignement et de recherche français ou étrangers, des laboratoires publics ou privés. 


\section{Adaptive Trajectory Tracking for Quadrotor MAVs in Presence of Parameter Uncertainties and External Disturbances}

\author{
Gianluca Antonelli ${ }^{\dagger}$, Elisabetta Cataldi $^{\dagger}$, Filippo Arrichiello ${ }^{\dagger}$, \\ Paolo Robuffo Giordano ${ }^{\ddagger}$, Stefano Chiaverini ${ }^{\dagger}$, and Antonio \\ Franchi?
}

\begin{abstract}
The paper presents an adaptive trajectory tracking control strategy for quadrotor Micro Aerial Vehicles (MAVs). The proposed approach, while maintaining the common assumption of an orientation dynamics faster than the translational one, removes the assumption of absence of external disturbances and of Geometric Center coincident with the Center of Mass. In particular, the trajectory tracking control law is made adaptive with respect to the presence of external forces and moments (e.g., due to wind) and to the uncertainty of parameters of the dynamic model, such as the position of the center of mass. A stability analysis is presented to analytically support the proposed controller, while numerical simulations are provided in order to validate its performance.
\end{abstract}

\section{INTRODUCTION}

Over the last years the robotics community experienced a substantial increase of interest in the Micro Aerial Vehicles (MAVs) field. In particular, quadrotor MAVs have become more and more widespread in the community as experimental platform for testing novel 3D planning, control and estimation schemes in real-world indoor and outdoor conditions. Indeed, in addition to being able to take-off and land vertically, quadrotors can reach high angular accelerations thanks to the relatively long lever arm between opposing motors.

The development of effective flight controllers and motion planning strategies has been one of the primary objectives in MAV research over the last decade, see e.g. [1]-[3]. Together with the constant improvements in the miniaturization of Micro Electro-Mechanical Systems and sensors (MEMS) and in the computational power of microcontrollers, this has led to impressive achievements by employing quadrotor MAVs as robotics platforms: planning and control for aggressive flight maneuvers [4] and collective control of multiple small- and micro-quadrotors [5], [6] are just a few examples.

Being the quadrotor an underactuated mechanical system (indeed, only four control inputs are available despite the six dimensions of its configuration), a common strategy is to control its 3D position and yaw angle, i.e., the so-called flat outputs for the system [7]. The addition of tilting propellers to the classical quadrotor model has nevertheless been recently explored in [8] in order to increase actuation capabilities and to gain full controllability of the 6-Degrees Of Freedom (DOF) of the quadrotor pose in space.

G. Antonelli, E. Cataldi, F. Arrichiello and S. Chiaverini are with University of Cassino and Southern Lazio, Via Di Biasio 43, 03043 Cassino, Italy \{antonelli, e.cataldi, f.arrichiello, chiaverini\}@unicas.it

$\ddagger$ P. Robuffo Giordano is with the CNRS at Irisa and Inria Rennes Bretagne Atlantique, Campus Beaulieu, 35042 Rennes Cedex, France prg@irisa. fr

2 A. Franchi is with LAAS-CNRS, Université de Toulouse, CNRS, Toulouse, France, afranchi@laas.fr
Robustness of the flight controller performance is a fundamental feature for any MAV application. Integral-based actions can be used to counteract external disturbances, such as wind and presence of small loads. Nevertheless, an adaptive/integral action may result in an additional disturbance when the nonlinearities of the model are not properly taken into account, see, e.g., [9] for analogous problems in the marine context. In this sense, at the best of our knowledge the only adaptive control for MAVs has been proposed in [10], [11].

The goal of this paper is to present a novel adaptive control scheme for quadrotor MAVs able to take into account the effects of constant exogenous forces and moments, as well as to cope with the presence of unknown dynamic parameters (e.g., the position of the CoM). Preliminary results of this work were presented in the papers [12] and [13]; in particular, the work [12] contains the stability analysis and numerical validation of the proposed adaptive control law, while [13] reports the experimental results. In this paper we extend these works by elaborating and discussing on the effects obtained when placing the origin of the body-fixed frame at the center of mass rather then at other more convenient locations, such as the quadrotor geometrical center. Moreover, here we present a more complete simulative case study considering both the cases of changing payload mass (such as during a transportation mission) and the presence of wind.

\section{MOdeling}

\section{A. Kinematics}

Let us define a frame $\Sigma_{b}, O_{b}-\boldsymbol{x}_{b} \boldsymbol{y}_{b} \boldsymbol{z}_{b}$ fixed to the MAV rigid body, from now on the body-fixed frame. The rigid body pose is described by its position and orientation with respect to a reference frame $\Sigma_{I}, O_{I}-\boldsymbol{x} \boldsymbol{y} \boldsymbol{z}$ that here is assumed as an inertial, North-East-Down, earth-fixed reference frame.

Let $\boldsymbol{\eta}_{1}=\left[\begin{array}{lll}x & y & z\end{array}\right]^{\mathrm{T}} \in \mathbb{R}^{3}$ be the position of $O_{b}$ expressed in $\Sigma_{I}$, and $\boldsymbol{\eta}_{2} \in \mathbb{R}^{3}$ be the vector collecting the set of body Euler-angle coordinates in $\Sigma_{I} \boldsymbol{\eta}_{2}=\left[\begin{array}{lll}\phi & \theta & \psi\end{array}\right]^{\mathrm{T}}$. Among the possible combinations, the roll, pitch and yaw angles are selected, i.e., the set of successive elementary rotations around $x, y$ and $z$ in $\Sigma_{I}$ [14]. The two vectors can be collected as $\boldsymbol{\eta}=\left[\begin{array}{ll}\boldsymbol{\eta}_{1}^{\mathrm{T}} & \boldsymbol{\eta}_{2}^{\mathrm{T}}\end{array}\right]^{\mathrm{T}}$ representing the overall six-dimensional position vector of the MAV.

The vectors $\dot{\boldsymbol{\eta}}_{1}$ and $\dot{\boldsymbol{\eta}}_{2}$ are time derivatives of $\boldsymbol{\eta}_{1}$ and $\boldsymbol{\eta}_{2}$ respectively (i.e., $\dot{\boldsymbol{\eta}}_{1}$ is the velocity of $O_{b}$ expressed in $\Sigma_{I}$ and $\dot{\boldsymbol{\eta}}_{2}$ is the derivative of the Euler-angles expressed in $\Sigma_{I}$ ).

Let's define $\boldsymbol{\nu}_{1}=\left[\begin{array}{lll}u & v & w\end{array}\right]^{\mathrm{T}}$ as the linear velocity of $O_{b}$ with respect to $O_{I}$, expressed in $\Sigma_{b}$, then it holds:

$$
\nu_{1}=\boldsymbol{R}_{I}^{b} \dot{\boldsymbol{\eta}}_{1}
$$

where $\boldsymbol{R}_{I}^{b}\left(\boldsymbol{\eta}_{2}\right)$ is the rotation matrix expressing the transformation from the $\Sigma_{I}$ to $\Sigma_{b}$.

Let's define $\boldsymbol{\nu}_{2}=\left[\begin{array}{lll}p & q & r\end{array}\right]^{\mathrm{T}}$ as the angular velocity of $\Sigma_{b}$ with respect to $\Sigma_{I}$ expressed in $\Sigma_{b}$. Following [14], the relation between the vectors $\dot{\boldsymbol{\eta}}_{2}$ and $\boldsymbol{\nu}_{2}$ can be expressed by the transformation matrix $T \in \mathbb{R}^{3 \times 3}$, i.e. :

$$
\boldsymbol{\nu}_{2}=\boldsymbol{T}\left(\boldsymbol{\eta}_{2}\right) \dot{\boldsymbol{\eta}}_{2}
$$

The two velocity vectors can be collected in the sixdimensional velocity vector $\boldsymbol{\nu}$ defined as $\boldsymbol{\nu}=\left[\begin{array}{ll}\boldsymbol{\nu}_{1}^{\mathrm{T}} & \boldsymbol{\nu}_{2}^{\mathrm{T}}\end{array}\right]^{\mathrm{T}}$. 


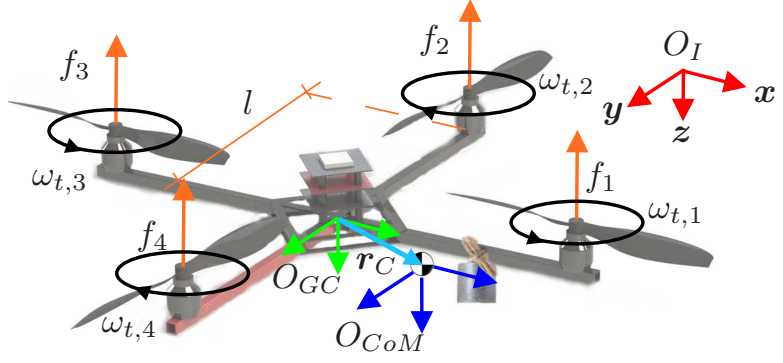

Fig. 1. Variables related to the actuation system with, in particular, the two possible placements for the origin of the body-fixed reference frame (Geometric Center (GC) or Center of Mass (CoM))

\section{B. Dynamics}

As done, among the others, in [1]-[5], we model the quadrotor dynamics using the equations of motion for a single 6-DOF rigid body, i.e. we do not consider here the effects of the spinning propellers on the main body dynamics. When placing the origin $O_{b}$ of the body-fixed reference frame, two choices are naturally possible: the Geometric Center (GC) of the quadrotor, i.e., the intersection between the two thrusters axes, or the Center of Mass (CoM) of the quadrotor structure (in Fig. 1 we mark as $O_{G C}$ as $O_{C o M}$ the respective two possible placements of $O_{b}$ ).

The rigid body dynamics of a quadrotor w.r.t. the body-fixed frame placed in the GC is given, in matrix form, by:

$$
M \dot{\nu}+C(\nu) \nu+g\left(\eta_{2}\right)=\tau+\tau_{W},
$$

where $\tau \in \mathbb{R}^{6}$ represents the generalized input forces acting on the vehicle. In detail, let us partition $\boldsymbol{\tau}$ as $\boldsymbol{\tau}=\left[\begin{array}{ll}\boldsymbol{\tau}_{1}^{\mathrm{T}} & \boldsymbol{\tau}_{2}^{\mathrm{T}}\end{array}\right]^{\mathrm{T}}$, where the vector $\boldsymbol{\tau}_{1}=\left[\begin{array}{lll}X & Y & Z\end{array}\right]^{\mathrm{T}}$ collects the linear forces acting on the rigid body expressed in the body-fixed frame, and the vector $\tau_{2}=\left[\begin{array}{lll}K & M & N\end{array}\right]^{\mathrm{T}}$ collects the moments acting on the rigid body expressed in the body-fixed frame.

The system inertia matrix $M \in \mathbb{R}^{6 \times 6}$ is constant, symmetric and positive definite, i.e., $\dot{M}=O, M=M^{\mathrm{T}}>O$. Its unique parametrization takes the form:

$$
\boldsymbol{M}=\left[\begin{array}{cc}
m \boldsymbol{I}_{3} & -m \boldsymbol{S}\left(\boldsymbol{r}_{C}^{b}\right) \\
m \boldsymbol{S}\left(\boldsymbol{r}_{C}^{b}\right) & \boldsymbol{I}_{O_{b}}
\end{array}\right]
$$

where $\boldsymbol{r}_{C}^{b}=\left[\begin{array}{lll}r_{C, x} & r_{C, y} & r_{C, z}\end{array}\right]^{T} \in \mathbb{R}^{3}$ is the center-ofmass position expressed in body-fixed frame, $\boldsymbol{I}_{3}$ is the $(3 \times 3)$ identity matrix, $\boldsymbol{I}_{O_{b}}$ is the inertia tensor expressed in the bodyfixed frame, $\boldsymbol{S}(\cdot)$ is the skew-symmetric matrix representing the cross product operator [14], and $m \in \mathbb{R}^{+}$is the total mass of the vehicle.

Explicit expressions for $C$ can be found in, e.g., [15].

The gravity generalized force $\boldsymbol{g} \in \mathbb{R}^{6}$, acting at the center of mass $\boldsymbol{r}_{C}^{b}$, is represented in body-fixed frame by:

$$
\boldsymbol{g}\left(\boldsymbol{\eta}_{2}\right)=-\left[\begin{array}{c}
m \boldsymbol{R}_{I}^{b} \boldsymbol{g}^{I} \\
m \boldsymbol{S}\left(\boldsymbol{r}_{C}^{b}\right) \boldsymbol{R}_{I}^{b} \boldsymbol{g}^{I}
\end{array}\right],
$$

where $\boldsymbol{g}^{I}=\left[\begin{array}{lll}0 & 0 & g\end{array}\right]^{\mathrm{T}} \mathrm{m} / \mathrm{s}^{2}$ and $g=9.81$. When placing the body-fixed frame at the CoM, this expression results simplified since the last three terms of the vector (i.e., the moment components) are null.
The term $\boldsymbol{\tau}_{W} \in \mathbb{R}^{6}$ represents external forces/moments acting on the vehicle due to disturbances such as wind. To this purpose, let define as $\gamma_{W} \in \mathbb{R}^{6}$ a vector of constant parameters; within a time interval in which the external disturbances can be considered as constant, their effect can be modeled in the vehicle-fixed frame as

$$
\boldsymbol{\tau}_{W}=\boldsymbol{\Phi}_{W}\left(\boldsymbol{\eta}_{2}\right) \gamma_{W}=\left[\begin{array}{cc}
\boldsymbol{R}_{I}^{b} & \boldsymbol{O}_{3 \times 3} \\
\boldsymbol{O}_{3 \times 3} & \boldsymbol{R}_{I}^{b}
\end{array}\right] \boldsymbol{\gamma}_{W}
$$

where the $(6 \times 6)$ regressor matrix $\boldsymbol{\Phi}_{W}$ expresses the force and moment coordinate transformation between the two frames.

By exploiting the linearity in the parameters, it is possible to rewrite (3) as:

$$
\Phi\left(\dot{\nu}, \nu, \boldsymbol{\eta}_{2}\right) \gamma=\tau
$$

where $\gamma \in \mathbb{R}^{16}$ collects the following dynamic parameters: the mass ( 1 param.), the first moment of inertia (3 param.), the inertia tensor (6 param.) and the disturbance $\gamma_{W}(6$ param.).

The first three rows of equation (7) can be rewritten with respect to the variables expressed in the inertial frame $\dot{\boldsymbol{\eta}}, \ddot{\boldsymbol{\eta}}$ by exploiting (1)-(2) and the corresponding time derivatives, according to the guidelines of robotics textbooks, e.g., [14][15]. In particular, on the base of the diagonal structure of the $M_{1,1}$ block of eq. (4), they can be decomposed in the terms $\boldsymbol{\Phi}_{x y} \in \mathbb{R}^{2 \times 16}$ and $\phi_{z} \in \mathbb{R}^{1 \times 16}$ denoting respectively the first two rows and third row of $\boldsymbol{\Phi}$ in (7) expressed in the inertial frame, i.e.,:

$$
\left[\begin{array}{c}
\left.\boldsymbol{\Phi}_{x y}\left(\begin{array}{c}
\ddot{x} \\
\ddot{y}
\end{array}\right], \ddot{\boldsymbol{\eta}}_{2}, \dot{\boldsymbol{\eta}}, \boldsymbol{\eta}_{2}\right) \\
\boldsymbol{\phi}_{z}\left(\ddot{z}, \ddot{\boldsymbol{\eta}} 2, \dot{\boldsymbol{\eta}}, \boldsymbol{\eta}_{2}\right)
\end{array}\right] \boldsymbol{\gamma}=\boldsymbol{R}_{b}^{I} \boldsymbol{\tau}_{1} .
$$

For the sake of space, in this work we will omit further details on the system dynamics. However, the controller presented in the following will intentionally only consider the parameters affecting the steady-state error; these will be listed case-by-case during the next developments.

\section{Thrusters}

Quadrotors are equipped with four thrusters aligned along the $\boldsymbol{z}_{b}$ axis placed at position $\boldsymbol{p}_{t, i}^{b} \in \mathbb{R}^{3}$, for $i=1, \ldots, 4$. Each thruster provides a force and a moment according to $f_{i}=b \omega_{t, i}^{2}, \quad \tau_{t, i}=d \omega_{t, i}^{2} \quad$ for $i=1, \ldots, 4$ where $\omega_{t, i}$ is the angular velocity of the $i$-th rotor, $b$ and $d$ are the thrust and drag coefficients. Figure 1 reports the common motor position, positive rotation directions and relevant variables. In this figure both the GC and CoM body-fixed frames are highlighted.

When calculating the input forces acting on the vehicle, for both GC- and CoM-centered models it holds

$$
\boldsymbol{\tau}_{1}=\left[\begin{array}{lll}
0 & 0 & Z_{c}
\end{array}\right]^{\mathrm{T}}=\left[\begin{array}{lll}
0 & 0 & \sum_{i=1}^{4}-f_{i}
\end{array}\right]^{\mathrm{T}} .
$$

The moment components $\tau_{2}$, on the other side, differ between the GC- and CoM-centered models since they are computed with respect to different axes. With a proper numbering of the thrusters, for the GC-centered model it holds:

$$
\boldsymbol{\tau}_{2}=\left[\begin{array}{lll}
l\left(f_{2}-f_{4}\right) & l\left(f_{1}-f_{3}\right) & \tau_{t, 1}-\tau_{t, 2}+\tau_{t, 3}-\tau_{t, 4}
\end{array}\right]^{\mathrm{T}}
$$


where $l$ is the arm length.

For the CoM-centered model, assuming $\boldsymbol{r}_{C}^{b}=$ $\left[\begin{array}{lll}r_{C, x} & r_{C, y} & r_{C, z}\end{array}\right]^{\mathrm{T}} \neq \mathbf{0}^{\mathrm{T}}$, the computation of the moments is not anymore as symmetric as in (10). Indeed, a value $r_{C, x} \neq 0$ and $r_{C, y} \neq 0$ introduces distortion effects along the pitch and roll directions, respectively. After few computations one has

$$
\begin{aligned}
& K=l\left(f_{2}-f_{4}\right)+r_{C, y}\left(f_{1}+f_{2}+f_{3}+f_{4}\right), \\
& M=l\left(f_{1}-f_{3}\right)-r_{C, x}\left(f_{1}+f_{2}+f_{3}+f_{4}\right),
\end{aligned}
$$

while the yaw torque $(\mathrm{N})$ is not affected by the CoM position. Due to the distributive property over the addition of the cross product, it is then easy to verify that

$$
\left(\boldsymbol{p}_{t, i}^{b}-\left[\begin{array}{c}
0 \\
0 \\
r_{C, z}
\end{array}\right]\right) \times\left[\begin{array}{c}
0 \\
0 \\
-f_{i}
\end{array}\right]=\boldsymbol{p}_{t, i}^{b} \times\left[\begin{array}{c}
0 \\
0 \\
-f_{i}
\end{array}\right] \forall i
$$

and thus the displacement along $\boldsymbol{z}_{b}$ of the center of gravity does not modify the moment contribution.

Since the dynamics of the low-level motor controller can be typically neglected with respect to the vehicle motion time scale, it is possible to express the mapping from the angular velocities to the force-torque acting on the vehicle as:

$$
\left[\begin{array}{l}
Z \\
K \\
M \\
N
\end{array}\right]=\boldsymbol{B}\left[\begin{array}{c}
\omega_{t, 1}^{2} \\
\omega_{t, 2}^{2} \\
\omega_{t, 3}^{2} \\
\omega_{t, 4}^{2}
\end{array}\right]=\boldsymbol{B} \boldsymbol{u}
$$

where $\boldsymbol{B}$ can assume the forms

$$
\begin{gathered}
\boldsymbol{B}_{G C}=\left[\begin{array}{cccc}
-b & -b & -b & -b \\
0 & b l & 0 & -b l \\
b l & 0 & -b l & 0 \\
d & -d & d & -d
\end{array}\right], \text { or } \\
\boldsymbol{B}_{C o M}=\left[\begin{array}{cccc}
-b & -b & -b & -b \\
b r_{C, y} & b\left(l+r_{C, y}\right) & b r_{C, y} & -b\left(l-r_{C, y}\right) \\
b\left(l-r_{C, x}\right) & -b r_{C, x} & -b\left(l+r_{C, x}\right) & -r_{C, x} \\
d & -d & d & -d
\end{array}\right] \text { (1) }
\end{gathered}
$$

depending on the choice of a GC or CoM-centered model, respectively. It is worth noting that, for the GC-centered model, matrix $\boldsymbol{B}$ does not depend on the CoM coordinates also in presence of a non-zero offset of the CoM with respect to the GC, i.e., when $\boldsymbol{r}_{C}^{b} \neq \mathbf{0}$.

\section{Discussion}

The reported analysis shows that the selection of where to place the origin of the body-fixed frame should be properly assessed when considering quadrotors with the CoM and the GC placed at different locations (as, e.g., when carrying external loads or because of imprecise calibration). Indeed, when opting for a CoM-centered model, the inertia matrix and gravity terms result simplified but, on the other hand, the computation of the thrust-generated moments shows a dependence on the (typically unknown) $\boldsymbol{r}_{C}$.

Conversely, the opposite choice of resorting to a GC-centered model solves the sensing part and simplifies the computation of the thrust-related moments but, on the other hand, introduces both an undesired coupling in the inertia matrix, and a moment contribution in the gravitational term. However, again, these effects cannot be compensated in the controller as they would require the knowledge of $\boldsymbol{r}_{C}$ that, on the contrary, is in most cases unknown.

Neglecting the displacement among the GC and the CoM in the feedback control law introduces a perturbation effect that can be evaluated as follows. Denoting with the subscript $c$ the force components elaborated by the feedback control assuming a GC-centered model, the actual forces components applied on the quadrotor in a CoM-centered model are

$$
\left[\begin{array}{c}
Z \\
K \\
M \\
N
\end{array}\right]=\boldsymbol{B}_{C o M} \boldsymbol{B}_{G C}^{-1}\left[\begin{array}{c}
Z_{c} \\
K_{c} \\
M_{c} \\
N_{c}
\end{array}\right]=\left[\begin{array}{cccc}
1 & 0 & 0 & 0 \\
-r_{C, y} & 1 & 0 & 0 \\
r_{C, x} & 0 & 1 & 0 \\
0 & 0 & 0 & 1
\end{array}\right]\left[\begin{array}{c}
Z_{c} \\
K_{c} \\
M_{c} \\
N_{c}
\end{array}\right] ;
$$

thus, a perturbation effect proportional to the offset is introduced in the $K$ and $M$ components; such perturbation might become significant for MAVs carrying a payload.

Therefore, the ability of being robust w.r.t. the CoM position by means of an online adaptive scheme is clearly beneficial for an effective flight control. The next section discusses a possible solution.

\section{QUADROTOR ADAPTIVE CONTROL}

We present here an adaptive control law for quadrotor position and yaw tracking based on the GC-centered model. The proposed solution consists of a set of interconnected control algorithms that allow to separately deal with horizontal/vertical position and yaw tracking, while managing the underactuated nature of the quadrotor structure exploiting the common assumption of an orientation dynamics significantly faster than the translational one. Desired force and torque are then used to compute the motors' square velocities $\boldsymbol{u}$ as

$$
\boldsymbol{u}=\boldsymbol{B}^{-1}\left[\begin{array}{llll}
Z_{c} & K_{c} & M_{c} & N_{c}
\end{array}\right]^{\mathrm{T}}
$$

where $\boldsymbol{B}=\boldsymbol{B}_{G C}$ as defined in (15).

\section{A. Altitude control}

By denoting $z_{d}$ as the desired altitude, let us define the error variables $\tilde{z}=z_{d}-z \in \mathbb{R}, s_{z}=\dot{\tilde{z}}+\lambda_{z} \tilde{z} \in \mathbb{R}$ with $\lambda_{z}>0$, and $\tilde{\gamma}_{z}=\gamma-\hat{\gamma}_{z} \in \mathbb{R}^{16}$, where $\gamma_{z}$ represents the combined contribution of the gravity and the vertical wind effects. The Lyapunov candidate function $V_{z}>0$ is

$$
V_{z}\left(s_{z}, \tilde{\gamma}_{z}\right)=\frac{m}{2} s_{z}^{2}+\frac{1}{2} \tilde{\gamma}_{z}^{\mathrm{T}} \boldsymbol{K}_{\gamma, z} \tilde{\gamma}_{z}
$$

with $\boldsymbol{K}_{\gamma, z}>\boldsymbol{O}$ being the adaptive gain.

The time derivative of $V\left(s_{z}, \tilde{\gamma}_{z}\right)$ is given by

$$
\dot{V}\left(s_{z}, \tilde{\gamma}_{z}\right)=s_{z}\left(m \ddot{z}_{d}-m \ddot{z}+m \lambda_{z} \dot{\tilde{z}}\right)-\tilde{\gamma}_{z}^{\mathrm{T}} \boldsymbol{K}_{\gamma, z} \dot{\hat{\gamma}}_{z}
$$

that, making $m \ddot{z}$ explicit from eq. (8), can be rewritten as

$$
\dot{V}\left(s_{z}, \tilde{\gamma}\right)=s_{z}\left(\phi_{z_{d}} \gamma-\left[\begin{array}{lll}
0 & 0 & 1
\end{array}\right] \boldsymbol{R}_{b}^{I} \boldsymbol{\tau}_{1}\right)-\tilde{\boldsymbol{\gamma}}_{z}^{\mathrm{T}} \boldsymbol{K}_{\gamma, z} \dot{\hat{\gamma}}_{z}
$$

in which $\phi_{z_{d}}=\phi_{z}\left(\ddot{z}_{d}+\lambda_{z} \dot{\tilde{z}}, \ddot{\boldsymbol{\eta}}_{2}, \dot{\boldsymbol{\eta}}, \boldsymbol{\eta}_{2}\right)$ and where $\phi_{z}$ is as defined in eq. (8). 
Assuming $\phi, \theta \neq \pm \pi / 2$, the scalar function $\dot{V}$ is made negative semidefinite by selecting

$$
\begin{aligned}
Z_{c} & =\frac{1}{\cos \phi \cos \theta}\left(\phi_{z_{d}} \hat{\gamma}_{z}+k_{v, z} s_{z}\right) \\
\dot{\hat{\gamma}}_{z} & =\boldsymbol{K}_{\gamma, z}^{-1} \phi_{z_{d}}^{\mathrm{T}} s_{z}
\end{aligned}
$$

yielding, after some algebra,

$$
\dot{V}_{z}\left(s_{z}, \tilde{\gamma}_{z}\right)=-k_{v, z} s_{z}^{2}
$$

We can now prove the system stability in a Lyapunov-like sense using Barbălat's Lemma [16]. Since

- $V_{z}\left(s_{z}, \tilde{\gamma}_{z}\right)$ is lower bounded;

- $\dot{V}_{z}\left(s_{z}, \tilde{\gamma}_{z}\right) \leq 0$;

- $\dot{V}_{z}\left(s_{z}, \tilde{\gamma}_{z}\right)$ is uniformly continuous;

then $\dot{V}_{z}\left(s_{z}, \tilde{\gamma}_{z}\right) \rightarrow 0$ as $t \rightarrow \infty$ and thus $s_{z} \rightarrow 0$. In particular, as usual when resorting to adaptive control techniques, one cannot prove asymptotic stability of the whole state since only boundedness of $\tilde{\gamma}_{z}$ can be guaranteed.

It is interesting to implement a simpler version of the controller aimed at compensating the sole persistent dynamic terms, i.e., those terms preventing a null steady-state error [9]:

$$
\begin{aligned}
Z_{c} & =\frac{1}{\cos \phi \cos \theta}\left(\hat{\gamma}_{z}+k_{v z} s_{z}\right) \\
\dot{\hat{\gamma}}_{z} & =k_{\gamma, z}^{-1} s_{z}
\end{aligned}
$$

in which $\gamma_{z} \in \mathbb{R}$ embeds the combined contribution of the gravity and the vertical wind effects.

\section{B. Horizontal position control}

By denoting $x_{d}, y_{d}$ the desired horizontal position, let us define $\tilde{\boldsymbol{\eta}}_{x y}=\left[\begin{array}{ll}x_{d}-x & y_{d}-y\end{array}\right]^{\mathrm{T}} \in \mathbb{R}^{2}, \boldsymbol{s}_{x y}=\dot{\tilde{\boldsymbol{\eta}}}_{x y}+\lambda_{x y} \tilde{\boldsymbol{\eta}}_{x y} \in \mathbb{R}^{2}$ with $\lambda_{x y}>0$, and $\tilde{\gamma}_{x y}=\gamma-\hat{\gamma}_{x y} \in \mathbb{R}^{16}$, where $\gamma_{x y}$ represents the wind horizontal effect. We consider the Lyapunov candidate function $V_{x y}>0$

$$
V_{x y}\left(\boldsymbol{s}_{x y}, \tilde{\boldsymbol{\gamma}}_{x y}\right)=\frac{1}{2} m \boldsymbol{s}_{x y}^{\mathrm{T}} \boldsymbol{s}_{x y}+\frac{1}{2} \tilde{\boldsymbol{\gamma}}_{x y}^{\mathrm{T}} \boldsymbol{K}_{\gamma, x y} \tilde{\boldsymbol{\gamma}}_{x y}
$$

where $\boldsymbol{K}_{\gamma, x y}>\boldsymbol{O}$ is the adaptive gain. The time derivative of $V_{x y}\left(\boldsymbol{s}_{x y}, \tilde{\gamma}_{x y}\right)$ is given by

$$
\dot{V}_{x y}=\boldsymbol{s}_{x y}^{\mathrm{T}}\left(\boldsymbol{\Phi}_{x y_{d}} \boldsymbol{\gamma}-\boldsymbol{R}_{z}^{\mathrm{T}}\left[\begin{array}{c}
c_{\phi} s_{\theta} \\
-s_{\phi}
\end{array}\right] Z_{c}\right)-\tilde{\boldsymbol{\gamma}}_{x y}^{\mathrm{T}} \boldsymbol{K}_{\gamma, x y} \hat{\boldsymbol{\gamma}}_{x y}
$$

with $\boldsymbol{\Phi}_{x y_{d}}=\boldsymbol{\Phi}_{x y}\left(\ddot{\boldsymbol{\eta}}_{d, x y}+\lambda_{x y} \dot{\tilde{\boldsymbol{\eta}}}_{x y}, \ddot{\boldsymbol{\eta}}_{2}, \dot{\boldsymbol{\eta}}, \boldsymbol{\eta}_{2}\right) \in \mathbb{R}^{2 \times 16}$ and and where $\boldsymbol{\Phi}_{x y}$ is as defined in eq. (8). Similarly to the analysis performed in the altitude control case, according to (9) the sole $Z_{c}$ component is considered as possible body-fixed force provided by the actuators. The scalar function $\dot{V}_{x y}$ can be made negative semidefinite by selecting the virtual inputs $\phi_{d}$ and $\theta_{d}$, for the orientation control so as to solve

$$
\begin{aligned}
{\left[\begin{array}{c}
c_{\phi_{d}} s_{\theta_{d}} \\
-s_{\phi_{d}}
\end{array}\right] } & =\frac{1}{Z_{c}} \boldsymbol{R}_{z}\left(\boldsymbol{\Phi}_{x y_{d}} \hat{\boldsymbol{\gamma}}_{x y}+k_{v, x y} \boldsymbol{s}_{x y}\right), \\
\dot{\hat{\gamma}}_{x y} & =\boldsymbol{K}_{\gamma, x y}^{-1} \boldsymbol{\Phi}_{x y_{d}}^{\mathrm{T}} \boldsymbol{s}_{x y} .
\end{aligned}
$$

In this case too, by considering only the persistent dynamic terms as in [9], the controller reduces to:

$$
\begin{aligned}
{\left[\begin{array}{c}
c_{\phi_{d}} s_{\theta_{d}} \\
-s_{\phi_{d}}
\end{array}\right] } & =\frac{1}{Z_{c}} \boldsymbol{R}_{z}\left(\hat{\boldsymbol{\gamma}}_{x y}+k_{v, x y} \boldsymbol{s}_{x y}\right) \\
\dot{\hat{\gamma}}_{x y} & =k_{\gamma, x y}^{-1} \boldsymbol{s}_{x y}
\end{aligned}
$$

where $\hat{\gamma}_{x y} \in \mathbb{R}^{2}$ represents the sole wind effect (supposed constant in the inertial frame), and $\phi_{d}$ and $\theta_{d}$ are sent to the orientation controller as the desired angles.

\section{Orientation control}

The orientation control receives as input the desired roll and pitch $\phi_{d}, \theta_{d}$ from the horizontal position control (see Section III-B) and the desired yaw $\psi_{d}$, and it outputs the torque components $\left[\begin{array}{lll}K_{c} & M_{c} & N_{c}\end{array}\right]^{\mathrm{T}}$.

When resorting to a GC-centered model, see (14)-(15), the moments generated by the controller can be transformed into the thruster angular velocities without the need of a known CoM position. On the other hand, at hovering, the vehicle is experiencing the moments from (5) computed for null roll and pitch, i.e., $\left.\boldsymbol{g}\right|_{\phi=0, \theta=0}=m g\left[\begin{array}{lll}r_{C, y} & -r_{C, x} & 0\end{array}\right]^{\mathrm{T}}$. This effect needs to be properly compensated and, thus, an integral or adaptive action is required to estimate the CoM position $\hat{r}_{C}$ in order to achieve null error at steady-state. The yaw dynamics, instead, appears to be decoupled from the remaining DOFs and it is not affected by the CoM displacement. The orientation control can be structured, as an example, as a set of independent Proportional-Derivative (PD) controllers of the different orientation components with gravity compensation:

$$
\begin{aligned}
K_{c} & =P D(\phi)+\hat{r}_{C, y} Z_{c}, \\
M_{c} & =P D(\theta)-\hat{r}_{C, x} Z_{c}, \\
N_{c} & =P D(\psi)
\end{aligned}
$$

with $\left[\begin{array}{l}\dot{\hat{r}}_{C, x} \\ \dot{\hat{r}}_{C, y}\end{array}\right]=k_{r_{C}}\left[\begin{array}{c}\theta_{d}-\theta \\ \phi-\phi_{d}\end{array}\right]$.

It is worth remarking that neither the altitude nor the yaw control loops are affected by the offset between CoM and GC; the convergence to a steady-state value for $Z_{c}$ can thus be assumed. In any case, roll and pitch control can be designed by considering the estimation error as an external, constant, disturbance and several adaptive control laws for attitude control may thus be used to the purpose.

\section{Simulations}

Numerical simulations in a wide range of operative conditions have been run in order to verify the effectiveness of the proposed adaptive controller. Here we report the results of numerical simulation in Matlab [17] environment gained with the sole reduced controller (i.e., the one ensuring a null steady-state error detailed in (24) and (28)) in order to prove that the persistent terms have been effectively compensated by resorting to a minimal set of five dynamic parameters; namely, $\left\{\gamma_{z}, \quad \gamma_{x y}, \quad r_{C, x}, \quad r_{C, y}\right\}$, where $\gamma_{z} \in \mathbb{R}$ represents the combined contribution of the gravity and the vertical wind effects, $\gamma_{x y} \in \mathbb{R}^{2}$ the wind horizontal effect, and $\boldsymbol{r}_{C}$ the center-of-mass position expressed in body-fixed frame.

In order to properly test the proposed adaptive control strategy, we consider the case of a quadrotor picking up and transporting an unknown object while being affected by a non-constant disturbance term due to wind. Table I reports the dynamic parameters of the simulated quadrotor (without considering the possibly transported object) and, when applicable, the initial values used by the adaptive controller. Table II 


\begin{tabular}{llll}
\hline & & simulated & initial estimate \\
\hline mass & $m$ & $1.50 \mathrm{~kg}$ & $1.49 \mathrm{~kg}$ \\
inertia & $\boldsymbol{I}_{O b}$ & $.025 \boldsymbol{I}_{3} \mathrm{~kg} \mathrm{~m}^{2}$ & $\begin{array}{l}\text { not used in the } \\
\text { reduced controller }\end{array}$ \\
length & $l$ & $30 \mathrm{~cm}$ & known \\
drag coeff. & $b$ & $1 \mathrm{Ns}^{2} / \mathrm{rad}^{2}$ & known \\
thrust coeff. & $d$ & $1 \mathrm{Nms}^{2} / \mathrm{rad}^{2}$ & known \\
center of mass & $\boldsymbol{r}_{C, b}$ & {$\left[\begin{array}{llll}5 & 3 & 0\end{array}\right]^{\mathrm{T}} \mathrm{cm}$} & {$\left[\begin{array}{lll}0 & 0 & 0\end{array}\right]^{\mathrm{T}} \mathrm{cm}$} \\
\hline
\end{tabular}

TABLE I

DATA USED IN THE SIMULATION

\begin{tabular}{lrrr}
\hline$\lambda_{z}$ & 1.1 & $\lambda_{x y}$ & 1.5 \\
$k_{v, z}$ & 3.0 & $k_{v, x y}$ & 2.0 \\
$k_{\gamma, z}$ & 1.3 & $k_{\gamma, x y}$ & 1.0 \\
& $k_{v, \phi \theta \psi}$ & 10.0 & \\
& $k_{p, \phi \theta \psi}$ & 40.0 & \\
& $k_{r C}$ & 5.0 & \\
\hline
\end{tabular}

TABLE II

GAINS USED IN THE SIMULATION

\begin{tabular}{lll}
\hline mass kg & $r_{C, x} \mathrm{~m}$ & $r_{C, y} \mathrm{~m}$ \\
\hline 0.5 & 0.070 & 0.050 \\
0.8 & 0.077 & 0.057 \\
1.0 & 0.082 & 0.062 \\
1.2 & 0.085 & 0.065 \\
\hline
\end{tabular}

TABLE III

MASSES OF THE ADDED OBJECT AND THE COM DISPLACEMENT. reports the control gains. The sampling time of the sensors and the controller have been set to $T=1 \mathrm{~ms}$.

We assume that the grasped object is a point mass of $0.5 \mathrm{~kg}$ and that the grasping point in the $O_{G C}$ frame is $\left[\begin{array}{lll}0.13 & 0.11 & 0.00\end{array}\right]^{\mathrm{T}} m$; thus, when the object is grasped, the position of the CoM of the system in the $O_{G C}$ frame becomes $\boldsymbol{r}_{C}=\left[\begin{array}{lll}0.07 & 0.05 & 0.00\end{array}\right]^{\mathrm{T}} m$.

The mission has been decomposed in the following time stages: $[0-10] \mathrm{s}$ : keep a hovering position in order to let the adaptive controller extinguish the initial transient; $[10-$ $20] \mathrm{s}$ : move toward the object position; $[20-40] \mathrm{s}$ : remain in hovering without carrying the object; $40 \mathrm{~s}$ : grasp the object; [40-80] s: remain in hovering while carrying the object; [8090] s: go back to the initial position; 90 s: release the object; $[90-100] \mathrm{s}$ : remain in hovering. Please notice that the system is wind affected in the time intervals $[30-40] \mathrm{s}$ and $[50-70] \mathrm{s}$.

Figure 2.a shows the components of the quadrotor position ( $x$-magenta, $y$-black, $z$-red) and its desired values (in gray). For sake of comparison, we performed the same mission using in one case the adaptive controller and in another case a non-adaptive controller that, instead, it is composed of a PID controller for both the vertical and horizontal control. Figure 2.b shows the norm of the position error for both the cases (the adaptive case in blue and the non-adaptive case in green) from which one can note the better performance of the proposed adaptive controller. It is worth noticing that the large errors in the $z$-direction around the time $40 \mathrm{~s}$ and $90 \mathrm{~s}$ are due respectively to the grasp and the release of the transported object that is simulated via an instantaneous change of the weight and of the position of the CoM.

Figures 2.c-d show in blue the roll and pitch angles and in gray the desired values obtained as outputs of the horizontal position control, see Sec. III-B; it can be noticed how the roll and pitch angles computed by the horizontal position control, in presence of constant input, converge to constant non-zero values in order to counteract for the external wind.

Figure 2.e shows the simulated and the estimated force along $z$-axis; as expected, when the quadrotor grasps the
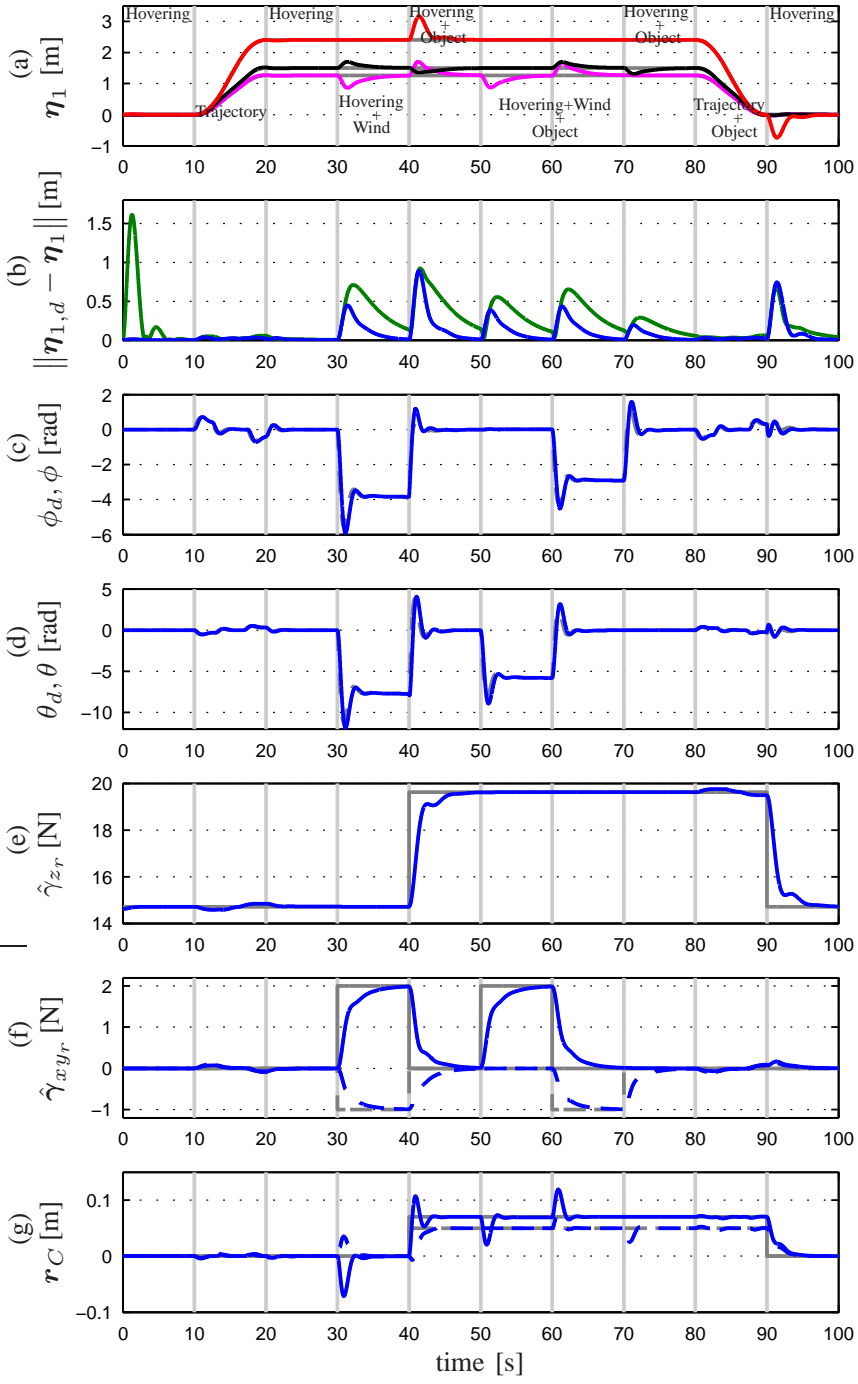

Fig. 2. Simulation results - (a) Quadrotor position along the different axis $x$-magenta, $y$-black, $z$-red; (b) Norm of the position error adaptive control (in blue) and the no-adaptive control (in green); (c) Desired (gray) and the effective values of the quadrotor roll; (d) desired (gray) and the effective value of the quadrotor pitch; (e) effective (gray) and estimated (blue) force along the $z$-axis; (f) effective (gray) and estimated (blue) forces along the $x, y$-axis, the solid line the $x$-axis and the dashed line the $y$-axis; (g) effective (gray) and estimated (blue) position of the CoM, the solid line the $x$-axis and the dashed line the $y$-axis.

object, the force along the $z$-axis changes due to the change of the total weight. One can also appreciate how the controller correctly estimates the total force.

The effective (gray) and estimated (blue) forces along the $x$-axis (solid-line) and $y$-axis (dashed-line) are shown in Figure 2.f; in this case, the controller ensures a null position error at steady-state. One can also appreciate that during the perturbation the $z$-axis remains almost constant.

Figure 2.g shows the effective (gray) and the estimated (blue) position of the CoM, where the $x$-axis components are the solid-lines and the $y$-axis components are the dashed-lines. It is possible to appreciate that the estimation of the CoM position is quite accurate even if the CoM position changes during the mission due to the grasp/release of the object. 


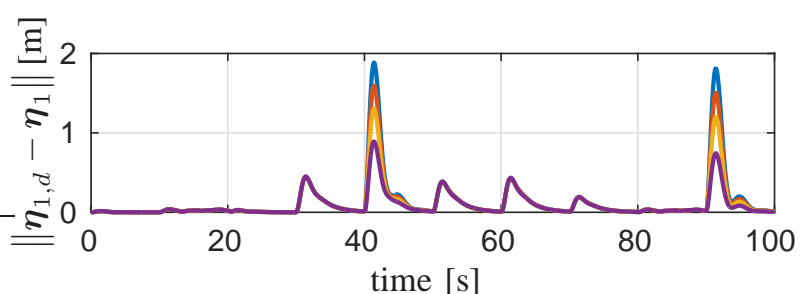

Fig. 3. Simulation results - Norm of the position error of the proposed control for four simulations with different mass attached, in blue $0.5 \mathrm{~kg}$, green $0.8 \mathrm{~kg}$. red $1.0 \mathrm{~kg}$ and cvan $1.2 \mathrm{~kg}$.

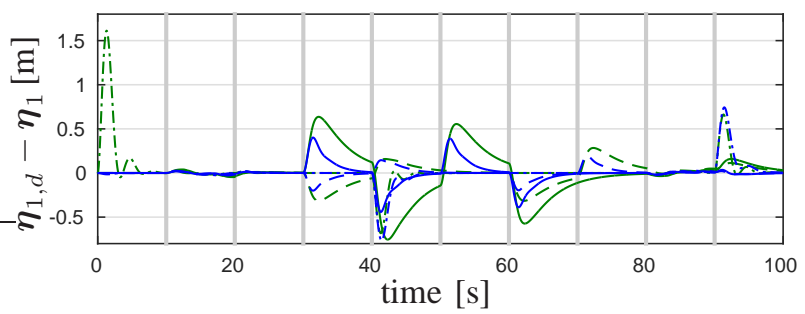

Fig. 4. Simulation results - In figure the position error for the adaptive control (blue) and the PID control (green) are reported, where the solid-line for the $x$-axes dot-line for the $y$-axis and the dot-point-line for the $z$-axis.

In order to evaluate the effectiveness of the proposed control law in different conditions, we repeated the simulations by considering different weights of the transported object. Table III reports, for the considered cases, the weights of the objects and the position of the CoM expressed in body-fixed frame. Figure 3 reports the norms of the position errors and it shows that, during the transient, the errors increase with the weight of the object, while in the state steady the errors converge to zero in all the cases.

In Figure 4 to better appreciate the performances of the proposed control the error of the proposed control (blue) and the PID control (green) are reported.

Results of an experimental validation of the proposed method can be found in [13] (details are here omitted for lack of space).

\section{CONCLUSION}

In this paper, an adaptive control scheme for quadrotor MAVs has been presented. By first discussing some modeling insights, we have shown how most of the literature resorts to a dynamic model, here labelled as CoM-centered model, that assumes the CoM coincident with the GC; when this assumption is not true, e.g. for MAVs carrying payloads or manipulators, neglecting the offset between CoM and GC implies some errors in the assumed placement of the onboard sensing and actuation system with respect to the CoM. This may cause unpredicted disturbance terms that are not easy to compensate since the exact CoM position is typically unknown. The adaptive control approach proposed in this paper is able to cope with an unknown CoM location, with uncertainties in MAV mass and with the possible presence of external disturbances. The proposed control approach is analytically supported by a stability analysis and its performance are validated via extensive numerical simulation results.

\section{ACKNOWLEDGMENTS}

The research leading to these results has received funding from the European Community's 7th FP under grant agreement No.287617 (IP project ARCAS) and from the European Community's Horizon 2020 Program under grant agreement No. 644271 (IP project AEROARMS).

\section{REFERENCES}

[1] N. Michael, D. Mellinger, Q. Lindsey, and V. Kumar, "The GRASP multiple micro-UAV testbed," Robotics and Automation Magazine, vol. 17, no. 3, pp. 56-65, 2010.

[2] S. Omari, M.-D. Hua, G. Ducard, and T. Hamel, "Hardware and software architecture for nonlinear control of multirotor helicopters," IEEE/ASME Transactions on Mechatronics, vol. 18, no. 4, pp. 1724-1736, 2013.

[3] M.-D. Hua, T. Hamel, P. Morin, and C. Samson, "Introduction to feedback control of underactuated vtol vehicles: A review of basic control design ideas and principles," IEEE Control System Magazine, vol. 33, no. 1, pp. 61-75, 2013.

[4] D. Mellinger, N. Michael, and V. Kumar, "Trajectory generation and control for precise aggressive maneuvers with quadrotors," in Proc. of the 2010 Int. Symposium on Experimental Robotics, 2010.

[5] A. Kushleyev, D. Mellinger, and V. Kumar, "Towards A Swarm of Agile Micro Quadrotors," in Proc. of the 2012 Robotics: Science and Systems, 2012.

[6] A. Franchi, C. Secchi, M. Ryll, H. H. Bülthoff, and P. Robuffo Giordano, "Shared control: Balancing autonomy and human assistance with a group of quadrotor uavs," Robotics and Automation Magazine, vol. 19, no. 3, pp. 57-68, 2012.

[7] M. Fliess, J. Lévine, P. Martin, and P. Rouchon, "Flatness and defect of nonlinear systems: Introductory theory and examples," International Journal of Control, vol. 61, no. 6, pp. 1327-1361, 1995.

[8] M. Ryll, H.-H. Bülthoff, and P. Robuffo Giordano, "A novel overactuated quadrotor uav: Modeling, control and experimental validation," IEEE Trans. on Control Systems Technology, vol. 23, no. 2, pp. 540-556, 2015.

[9] G. Antonelli, "On the use of adaptive/integral actions for 6-degrees-offreedom control of autonomous underwater vehicles," IEEE Journal of Oceanic Engineering, vol. 32, no. 2, pp. 300-312, April 2007.

[10] I. Palunko and R. Fierro, "Adaptive control of a quadrotor with dynamic changes in the center of gravity," in Proceedings 18th IFAC World Congress, vol. 18, no. 1, Milan, I, August 2011, pp. 2626-2631.

[11] I. Palunko, P. Cruz, and R. Fierro, "Agile load transportation. Safe and efficient load manipulation with aerial robots," Robotics and Automation Magazine, pp. 69-79, 2012.

[12] G. Antonelli, F. Arrichiello, S. Chiaverini, and P. Robuffo Giordano, "Adaptive trajectory tracking for quadrotor MAVs in presence of parameter uncertainties and external disturbances," in Proceedings 2013 IEEE/ASME International Conference on Advanced Intelligent Mechatronics, Wollongong, AU, July 2013.

[13] G. Antonelli, E. Cataldi, P. Robuffo Giordano, S. Chiaverini, and A. Franchi, "Experimental validation of a new adaptive control scheme for quadrotors MAVs," in 2013 IEEE/RSJ International Conference on Intelligent Robots and Systems, Tokyo, JP, Nov. 2013, pp. 3496-3501.

[14] B. Siciliano, L. Sciavicco, L. Villani, and G. Oriolo, Robotics: modelling, planning and control. Springer Verlag, 2009.

[15] T. Fossen, Marine Control Systems: Guidance, Navigation and Control of Ships, Rigs and Underwater Vehicles. Trondheim, Norway: Marine Cybernetics, 2002.

[16] H. Khalil, Nonlinear Systems, 2nd ed. Upper Saddle River, New Jersey: Prentice-Hall, 1996.

[17] MATLAB, version 7.10.0 (R2010a). Natick, Massachusetts: The MathWorks Inc., 2010. 